\title{
$\beta$-Adrenergic Blockade during Memory Retrieval in Humans Evokes a Sustained Reduction of Declarative Emotional Memory Enhancement
}

\author{
Marijn C. W. Kroes, ${ }^{1,2}$ Bryan A. Strange, ${ }^{1}$ and Raymond J. Dolan ${ }^{1}$ \\ ${ }^{1}$ Wellcome Trust Centre for Neuroimaging, Institute of Neurology, London WC1N 3BG, United Kingdom, and ${ }^{2}$ Donders Institute for Brain, Cognition and \\ Behavior, Centre for Cognitive Neuroimaging, Radboud University, 6500 HB Nijmegen, The Netherlands
}

\begin{abstract}
Memory enhancement for emotional events is dependent on amygdala activation and noradrenergic modulation during learning. A potential role for noradrenaline (NE) during retrieval of emotional memory is less well understood. Here, we report that administration of the $\beta$-adrenergic receptor antagonist propranolol at retrieval abolishes a declarative memory enhancement for emotional items. Critically, this effect persists at a subsequent $24 \mathrm{~h}$ memory test, in the absence of propranolol. Thus, these findings extend our current understanding of the role of NE in emotional memory to encompass effects at retrieval, and provide face validity to clinical interventions using $\beta$-adrenergic antagonists in conjunction with reactivation of unwanted memories in anxiety-related disorders.
\end{abstract}

\section{Introduction}

Enhanced episodic memory for emotional events (Christianson, 1992; Cahill et al., 1995) is dependent on the amygdala (LeDoux, 2000). During initial acquisition or consolidation of an emotional memory, the amygdala is thought to upregulate neuronal processing in the hippocampus (McGaugh et al., 2002; Richardson et al., 2004) through a $\beta$-adrenergic mechanism (Cahill and McGaugh, 1998; McGaugh, 2004; Strange and Dolan, 2004). $\beta$-Adrenergic blockade with the $\beta_{1} \beta_{2}$-antagonist propranolol selectively impairs long-term human episodic memory for emotionally arousing material without affecting memory for neutral material when administered before learning (Cahill et al., 1994; Strange et al., 2003). This widely accepted model of noradrenergic influences on human emotional memory, by focusing on encoding and consolidation (Cahill and McGaugh, 1998), implicitly suggests that the role of noradrenaline (NE) does not extend to retrieval of emotional memories. Providing evidence for a role of NE in retrieval would therefore add considerably to a broader understanding of the nature of emotional influences on memory.

Although adaptive to survival, emotional memory enhancement may also have pathological consequences, as in anxietyrelated disorders such as post-traumatic stress disorder (PTSD) (Ressler and Mayberg, 2007). Once consolidated, memories are thought to be relatively insensitive to disruption or additional modulation (McGaugh, 2000), rendering pharmacological treat-

\footnotetext{
Received Nov. 4, 2009; accepted Dec. 7, 2009.

This work was supported by a Wellcome Trust Programme Grant (R.J.D.). M.C.W.K. and B.A.S. acquired and analyzed data. M.C.W.K., B.A.S., and R.J.D. designed the study and wrote this manuscript.

The authors declare no competing financial interests.

Correspondence should be addressed to Bryan A. Strange, Wellcome Trust Centre for Neuroimaging, 12 Queen Square, London WC1N 3BG, UK. E-mail: bstrange@fil.ion.ucl.ac.uk.

DOI:10.1523/JNEUROSCI.5469-09.2010

Copyright $\odot 2010$ the authors $\quad 0270-6474 / 10 / 303959-05 \$ 15.00 / 0$
}

ments long after the traumatic event therapeutically ineffective. However, if neuromodulatory effects on emotional memory after consolidation were evident, then this would provide a potential therapeutic approach for attenuating the retrieval of unwanted, established, emotional memories. As such, the application of NE antagonists might, for example, reduce the occurrence of intrusive memories such as flashbacks in PTSD. Identifying a role for NE during emotional memory retrieval would therefore be of considerable clinical significance.

Currently, the role of NE at retrieval is unclear. Human brain imaging during emotional memory retrieval demonstrates activation of the locus ceruleus (LC) (Sterpenich et al., 2006), the origin of noradrenergic forebrain projections (Pickel et al., 1974; Fallon et al., 1978). Other studies demonstrate amygdala engagement during similar emotional memory retrieval tasks (Maratos et al., 2001; Smith et al., 2004, 2006). However, human psychopharmacological data fail to demonstrate blockade of enhanced emotional retrieval by propranolol given at recall (de Quervain et al., 2007; Tollenaar et al., 2009). This contrasts with observations in rodents that propranolol, given before retrieval of contextual conditioned fear, attenuates the fear response (Murchison et al., 2004). One possible explanation for this discrepancy is that the memory paradigm used in previous human studies (de Quervain et al., 2007; Tollenaar et al., 2009) did not evoke a robust emotional memory enhancement. Thus, the absence of consistent and robust emotional memory enhancements in these studies indicates a relatively marginal contribution of the adrenergic system in these tasks, such that an effect of propranolol could not be detected. Therefore, we conducted a double-blind, placebocontrolled experiment testing the effect of propranolol on cued recall of emotionally aversive verbal stimuli, using a stimulus set known to cause a marked emotional memory enhancement effect that is also known to be dependent on the amygdala and NE (Strange et al., 2003; Strange and Dolan, 2004). 
Briefly, on day 1 subjects encoded neutral control nouns (C) with occasional nouns being emotionally aversive (E nouns) or perceptually distinct (P nouns) (Strange et al., 2003) (Fig. 1A). On day 2, subjects were administered either propranolol (40 mg) or placebo and $90 \mathrm{~min}$ later engaged in a cued-recall test (Fig. $1 B)$. To rule out the possibility that any modulation of emotional memory retrieval by propranolol is restricted to testing in the presence of drug, we again tested subjects $24 \mathrm{~h}$ later, in the absence of placebo or drug, using the cued-recall test. We demonstrate enhanced memory for $\mathrm{E}$ relative to $\mathrm{C}$ nouns in the placebo group on both day 2 and day 3. Administration of propranolol at retrieval abolishes emotional memory enhancement on day 2 , an effect that is present at least $24 \mathrm{~h}$ later on day 3 .

\section{Materials and Methods}

Twenty-four right-handed native English speakers with normal/corrected to normal vision, without cardiorespiratory, neurological, or psychiatric history participated in the study. The placebo and propranolol groups each comprised 12 subjects, 7 males and 5 females, respectively, with a mean age of 24.4 years (range, 19-34). One male subject in the propranolol group only completed day 1 and day 2 of the experiment. All subjects gave informed consent, and the study had full ethics approval. The experiment was conducted on 3 consecutive days, with each session at the same time of day and in the same testing room for a given subject.

Day 1. Blood pressure (BP) was measured and an electrocardiogram acquired for each participant on arrival. During encoding, subjects viewed 360 nouns presented visually in uppercase at a rate of one noun every $3 \mathrm{~s}$ (stimulus duration, $1 \mathrm{~s}$ ). The nouns consisted of 300 neutral nouns all presented in the same font, 30 nouns were emotionally aversive oddballs (E), presented in the same font as the neutral nouns, and 30 nouns were perceptual oddballs $(\mathrm{P})$, presented in different fonts. E nouns were selected from the set used in our previous studies (Strange et al., 2003; Richardson et al., 2004). Subjects engaged in a deep encoding task (Craik and Lockhart, 1972), making a push button response to indicate whether the noun described a living or nonliving entity. Although we have previously shown emotional memory enhancement to be more pronounced after shallow encoding (Strange et al., 2003), a deep encoding task was chosen to ensure adequate memory performance on day 2 and day 3 . Word presentation was randomized and oddballs were randomly presented after four to six (mean, five) neutral words. Words were "stem-unique" in that the first three letters were different for each of the 360 words. A total of 120 words served as controls (C), with the constraint that these controls were more than one word before or after a given oddball.

Day 2. BP was again measured and subjects were then administered either an oral dose of $40 \mathrm{mg}$ of propranolol or placebo (vitamin C) in a double-blind experimental design. After 80 min, subjects filled out the State form of the State-Trait Anxiety Inventory (STAIS) (Spielberger et al., 1983) and the Beck Depression Inventory-II (BDI) (Beck et al., 1996). Subjects then performed a surprise memory task on the words encoded on day 1 . In view of the kinetics of the peak plasma concentration (1-2h) of propranolol, the memory task started 90 min after drug administration. Randomly selected word stems (first three letters) for 240 of the 360 nouns encoded on day 1 were presented visually in uppercase in random order. Of these, 200 were neutral, 20 were emotional oddballs, and 20 were perceptual oddballs. All nouns were presented in the same font, including nouns that were perceptually distinct on day 1 (i.e., P nouns). Subjects were instructed to complete the stems to make a word encoded on day 1. Presentation of stimuli was continuous and stems were presented every $4 \mathrm{~s}$ with a stimulus duration of $1 \mathrm{~s}$. We used a cued-recall test of retrieval to increase recall performance from a large study list.

Day 3. This followed the same procedure as day 2 except that no drug was administered on day 3 . Furthermore, 360 stems were presented; the 240 presented on day 2 plus stems pertaining to the remaining 100 neutral words, 10 emotional oddballs, and 10 perceptual oddballs from day 1 that had not been presented on day 2 .

\section{Results}

Propranolol did not affect cued recall of control nouns (Fig. 2A). A group ("placebo"; "propranolol") by day (day 2; day 3) $2 \times 2$ repeated-measures ANOVA on $C$ noun recall revealed no main effects of group $\left(F_{(1,10)}=0.706 ; p=0.420\right)$ or day $\left(F_{(1,10)}=\right.$ $2.124 ; p=0.176)$ and no group by day interaction $\left(F_{(1,10)}=\right.$ $0.381 ; p=0.551)$. No memory enhancement was detected for perceptual oddballs (see supplemental material, available at www.jneurosci.org), and as such remaining analyses were restricted to emotional versus neutral nouns. Importantly, recall performance for all words was significantly above chance as indicated in a separate experimental baseline cue completion group (see supplemental material, available at www.jneurosci.org).

In our critical comparison, we tested for the effect of drug manipulation on $\mathrm{E}$ noun memory relative to subject-specific $\mathrm{C}$ noun recall (Fig. 2A). A group (placebo; propranolol) by day (day 2 ; day 3 ) by noun type (emotional; control) $2 \times 2 \times 2$ repeatedmeasures ANOVA revealed a significant main effect of group $\left(F_{(1,10)}=9.491 ; p=0.012\right)$ and noun type $\left(F_{(1,10)}=8.461 ; p=\right.$ $0.016)$, and a significant group by noun type interaction $\left(F_{(1,10)}=\right.$ 
A

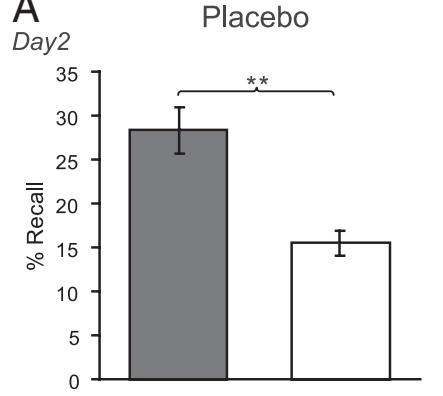

Day3
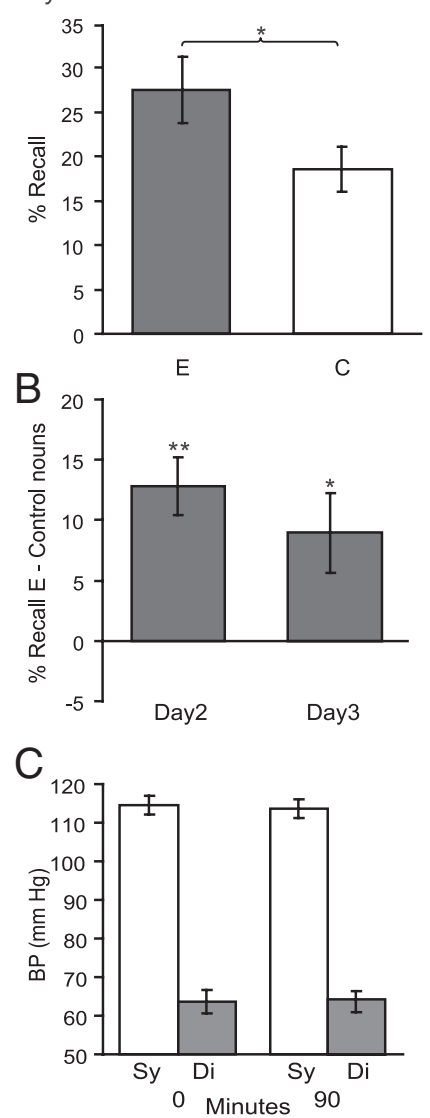

Propranolol

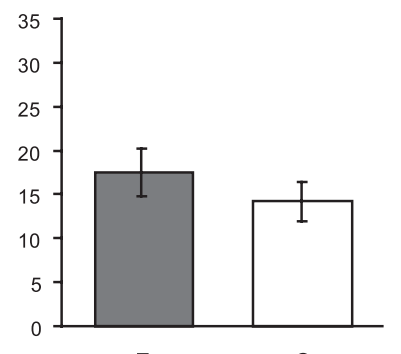

E
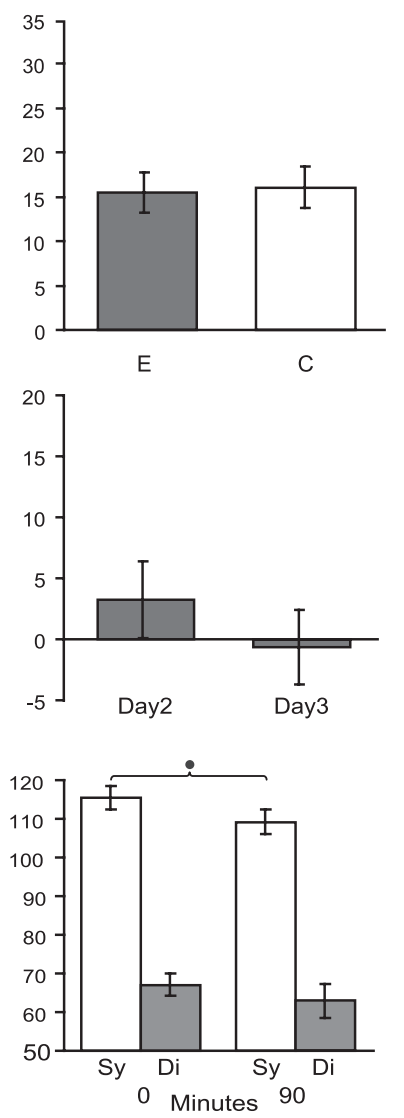

Figure 2. Propranolol at retrieval causes a sustained reduction of emotional memory enhancement. $\boldsymbol{A}$, Cued recall (percentage hits) on day 2 (top) and day 3 (bottom) of emotional nouns (E) (gray) and control nouns (C) for the placebo (left column) and propranolol (right column) groups. Drug manipulation before retrieval on day 2 causes a significant reduction of $E$ relative to $($ recall on day 2 , which is sustained on day 3 in the absence of drug. $\boldsymbol{B}$, Cued recall (percentage hits) of emotional nouns minus subject-specific control noun recall on day 2 and day 3 for the placebo (left column) and propranolol (right column) groups. C, Systolic (Sy) and diastolic (Di) BP (in millimeters mercury) before placebo (left column) or propranolol (right column) administration ( $0 \mathrm{~min}$ ) and after $90 \mathrm{~min}$. No baseline difference in systolic or diastolic $\mathrm{BP}$ is present between groups. The propranolol group displays a significant reduction in systolic BP 90 min after application. Error bars indicate SEM. ${ }^{* *} p<0.001$, two-tailed; ${ }^{*} p<0.05$, two-tailed; $\bullet p<0.05$, one-tailed.

13.174; $p=0.005)$, with no other significant main effects or interactions.

Planned comparisons confirmed that, on day 2, the placebo group showed enhanced recall for emotional nouns relative to control nouns $\left(t_{(11)}=5.365 ; p<0.001\right.$, two-tailed), whereas no emotional memory enhancement was evident in the propranolol group $\left(t_{(11)}=1.044 ; p=0.319\right.$, two-tailed) (Fig. $2 B$ ). On day 3 , the placebo group continued to show enhanced recall for emo-

tional relative to control nouns $\left(t_{(11)}=2.702 ; p=0.021\right.$, twotailed). Critically, subjects who had received propranolol on the previous day persist in showing an absent emotional memory enhancement on day $3\left(t_{(11)}=-0.202 ; p=0.844\right.$, two-tailed) (Fig. $2 B$ ). Directly comparing E noun recall between the placebo and propranolol group revealed reduced memory scores for the propranolol group on day $2\left(t_{(22)}=2.900 ; p=0.008\right.$, two-tailed $)$ and on day $3\left(t_{(21)}=2.701 ; p=0.013\right.$, two-tailed $)$. In both groups, the E nouns remembered on day 2 tended to be the same as those remembered on day 3 (see supplemental Results, Fig. S1, available at www.jneurosci.org as supplemental material).

Cued-recall performance for perceptual nouns, as well as for nouns cued only on day 3 , is given in supplemental Fig. S2 (available at www.jneurosci.org as supplemental material). For the items that had not been presented on day 2, drug manipulation did not affect mean recall of control nouns on day $3\left(t_{(21)}=\right.$ $-1.177 ; p=0.255$, two-tailed). Critically, no recall differences between the drug groups were found for the new emotional nouns $\left(t_{(21)}=0.153 ; p=0.880\right.$, two-tailed).

On day 2, there was no baseline systolic BP differences between groups $\left(t_{(21)}=-0.223 ; p=0.826\right.$, two-tailed). In line with previous findings (Tollenaar et al., 2009), we observe a drop in systolic BP in the propranolol group $\left(t_{(20)}=1.842 ; p=0.04\right.$, one-tailed) after $90 \mathrm{~min}$, but no drop in diastolic BP (Fig. 2C). Note that at both time points BP measurements for a different placebo subject was lost because of equipment failure.

\section{Discussion}

Our data provide a clear demonstration of a critical role for NE in the retrieval of emotional memory. We show that $\beta$-adrenergic blockade by propranolol at retrieval abolishes the declarative memory enhancement for emotional items. Second, we show that $24 \mathrm{~h}$ after $\beta$-adrenergic blockade at retrieval, a sustained reduction of emotional item recall is still observed in a second retrieval test in the absence of propranolol. Our findings provide support for the possibility of lasting attenuation of emotional memory in therapeutic contexts by pharmacological interventions at retrieval.

Our finding of a role for NE in retrieval of emotional memory speaks directly to the discrepancy between neuroimaging data, which implicate the NE system in emotional memory retrieval (Sterpenich et al., 2006), and human psychopharmacological studies, which report no effect of propranolol administration at retrieval on the enhancing effect of emotion on memory (de Quervain et al., 2007; Tollenaar et al., 2009). The latter negative findings might be explained by an absence of robust memory enhancement for emotional items in these tasks, possibly because of the presentation of equal numbers of neutral and emotional items and/or the presentation of emotional items of both positive and negative valence (Tollenaar et al., 2009). The use of equal numbers of emotional and neutral items in these previous studies may have resulted in rapid amygdala habituation (Fischer et al., 2003), attenuating the memory enhancement process. In the oddball task used here, the amygdala response to emotional items has been shown not to habituate over repeated exposure (Strange and Dolan, 2001). Furthermore, valence can enhance memory for low-arousing items via prefrontal-based organizational strategies during retrieval that are independent of the amygdala and noradrenergic modulation (Kensinger and Corkin, 2004; Mickley Steinmetz and Kensinger, 2009). Indeed, we note a suggestion (Tollenaar et al., 2009) that a lack of an effect of propranolol might be attributable to a nondependence of their task on the amygdala and/or NE. 
Animal data indicate that a role for NE in emotional memory retrieval is time specific. Emotional memory retrieval is affected by noradrenergic manipulation at $2 \mathrm{~h}$, but not $1 \mathrm{~h}$, after learning, and this effect is absent if the study-test interval is extended to $4 \mathrm{~d}$ (Murchison et al., 2004). The modulation of emotional memory by propranolol in humans may, therefore, also be time dependent. Thus, a second explanation for the discrepancy between our findings and a previous study (Tollenaar et al., 2009) might reflect the difference in study-test interval. This previous study, which found no effect of propranolol administration on emotional memory retrieval, tested retrieval 1 week after initial learning (Tollenaar et al., 2009) compared with the delay interval used here of $24 \mathrm{~h}$. It will be of interest to test whether the effect of propranolol observed in the current study is still observed if memory is tested $>4 \mathrm{~d}$ after learning.

A critical question pertains to the neurobiological basis for the dependency of emotional episodic memory retrieval on the $\beta$-adrenergic system. The current data do not directly address this, but potential explanations include the requirement for a cue-evoked phasic rise in NE (Aston-Jones and Cohen, 2005) such that there is congruence between NE signals at encoding and retrieval (Morris et al., 1977). Alternatively, the emotional memory trace may be dependent on tonic noradrenergic input (Murchison et al., 2004; Zhang et al., 2005) such that engagement of $\beta$-adrenergic receptors is essential for retrieval. It is conceivable that tonic NE input is no longer required after completion of systems level consolidation (Marr, 1970), which concords with a time-limited role of $\mathrm{NE}$ in emotional memory retrieval derived from animal data (Murchison et al., 2004).

In terms of a neuroanatomical basis for the effects we observe, neuroimaging studies demonstrate amygdala involvement in emotional memory retrieval. Amygdala activity has been reported during retrieval of neutral stimuli previously encoded in an emotional context (Maratos et al., 2001; Phelps et al., 2001; Smith et al., 2004; Fenker et al., 2005). The amygdala may directly support emotional memory retrieval or could achieve this through hippocampal modulation. Increased hippocampalamygdala coupling during retrieval of information encoded in an emotional context has been demonstrated in human (Smith et al., 2006) and rodent (Seidenbecher et al., 2003) studies. We suggest, therefore, that the effects we observe result from a propranolol-evoked disruption of amygdala responses, or hippocampal-amygdala coupling.

Animal studies have provided evidence for a more general role of NE in retrieval (Sara and Devauges, 1988, 1989; Devauges and Sara, 1991; Murchison et al., 2004). Specifically, increasing NE activity through pharmacological manipulation or by stimulation of the LC can restore forgotten spatial memory in rats (Sara and Devauges, 1988, 1989), via engagement of $\beta$-adrenergic receptors (Devauges and Sara, 1991). Thus, these studies extend the influence of $\mathrm{NE}$ at retrieval to include nonemotional memories. In contrast to this, the effect of propranolol in our task did not extend to cued recall of neutral words or items that were perceptually distinct at encoding (see supplemental material, available at www.jneurosci.org), which may reflect the essential nonverbal nature of animal tasks or effective central propranolol concentrations in human versus animal studies.

Our second main finding is that $\beta$-adrenergic blockade at retrieval leads to a sustained reduction of emotional memory retrieval $24 \mathrm{~h}$ later. The neurobiological basis for this effect could reflect a number of processes including a disruption of secondary encoding (Nadel and Land, 2000), a disruption of reconsolidation (Nader et al., 2000; Debiec and Ledoux, 2004), or an impair- ment of lingering consolidation (Dudai and Eisenberg, 2004). Secondary encoding is the process whereby, during every retrieval episode, retrieved mnemonic information, together with incoming sensory information, is stored as a novel memory trace (Nadel and Moscovitch, 1997). This results in the accumulation of multiple, related, mnemonic traces of a single episode within a slightly altered neuronal and experiential context, which together aid the retrieval of an episode (Nadel and Moscovitch, 1997). Thus, blocking enhanced recall of emotional stimuli on day 2 with propranolol may have prevented formation of a novel emotional memory trace, expressed as absent emotional memory enhancement in this group on day 3.

Alternatively, impaired reconsolidation may account for, at least in part, the memory effects observed on day 3. Reconsolidation refers to the idea that, on reactivation, memories return to a labile state and are once again sensitive to disruption (Nader et al., 2000). It is also known that reconsolidation of emotional memories is disrupted by blocking NE within the amygdala (Debiec and Ledoux, 2004). The long-term memory impairment we observe on day 3 could be attributed to propranolol having blocked reconsolidation of the episodic enhancement process. As such, the mechanisms mediating the enhancement of memory for emotional items might not have been expressed. Typically, in reconsolidation experiments, memories need to be reactivated to return to a labile state. However, in the case of amygdala processing of fear information, there is evidence that fear memories can undergo reconsolidation even if not expressed (Duvarci et al., 2006). Thus, propranolol might have impaired the reconsolidation of emotional items not remembered on day 2, reducing their chance of memory retrieval on day 3 .

The third possible explanation for our effects on day 3 is propranolol-induced impaired lingering consolidation. The lingering consolidation hypothesis posits that iterations of reactivation and stabilization of memory traces are an essential feature of consolidation, which, in contradistinction to standard models of consolidation, is not time limited (Dudai and Eisenberg, 2004). The memory effects observed on day 3 could, therefore, be attributed to a disruption of ongoing consolidation. However, an argument against this explanation is the absence of any difference between propranolol and placebo groups on memory for emotional items on day 3 that were not cued on day 2. Thus, the neurobiological mechanisms underlying our second main finding, that emotional memory impairment evoked by propranolol on day 2 persists to day 3 , are likely to be a failure of secondary encoding, impaired reconsolidation, or a combination of these processes. Thus, blocking enhanced recall of emotional stimuli on day 2 with propranolol may have prevented formation of a novel emotional memory trace, expressed as absent emotional memory enhancement in this group on day 3 .

The efficacy of pharmacological prevention of the acquisition of memories for traumatic experiences is limited by practicality (Pitman et al., 2002; Vaiva et al., 2003). The possibility of pharmacologically modulating established emotional memory at retrieval we demonstrate here supports a potential therapeutic approach of attenuating unwanted established emotional memories. Our data support recent clinical findings that propranolol administration to PTSD patients, after memory reactivation, reduces physiological responses to a subsequent script-driven trauma imagery session (Brunet et al., 2008). We extend these findings of post-reactivation modulation of implicit measures of emotional memory by propranolol (Brunet et al., 2008; Kindt et al., 2009) by demonstrating specific attenuation of retrieval of emotional episodic memory, without an impact on neutral mem- 
ory. Thus, we provide a theoretical justification for the potential application of propranolol in reducing retrieval of traumatic memories that justify studies of clinical interventions with $\beta$-adrenergic antagonists during reactivation of traumatic memory in anxiety-related disorders.

\section{References}

Aston-Jones G, Cohen JD (2005) An integrative theory of locus coeruleusnorepinephrine function: adaptive gain and optimal performance. Annu Rev Neurosci 28:403-450.

Beck AT, Steer RA, Brown GK, eds (1996) Manual for the Beck Depression Inventory-II. San Antonio, TX: Psychological Corporation.

Brunet A, Orr SP, Tremblay J, Robertson K, Nader K, Pitman RK (2008) Effect of post-retrieval propranolol on psychophysiologic responding during subsequent script-driven traumatic imagery in post-traumatic stress disorder. J Psychiatr Res 42:503-506.

Cahill L, McGaugh JL (1998) Mechanisms of emotional arousal and lasting declarative memory. Trends Neurosci 21:294-299.

Cahill L, Prins B, Weber M, McGaugh JL (1994) $\beta$-Adrenergic activation and memory for emotional events. Nature 371:702-704.

Cahill L, Babinsky R, Markowitsch HJ, McGaugh JL (1995) The amygdala and emotional memory. Nature 377:295-296.

Christianson S, ed (1992) The handbook of emotion and memory: research and theory. Hillsdale, NJ: Lawrence Erlbaum Associates.

Craik FI, Lockhart RS (1972) Levels of processing: a framework for memory research. J Verbal Learn Verbal Behav 11:671-684.

Debiec J, Ledoux JE (2004) Disruption of reconsolidation but not consolidation of auditory fear conditioning by noradrenergic blockade in the amygdala. Neuroscience 129:267-272.

de Quervain DJ, Aerni A, Roozendaal B (2007) Preventive effect of $\beta$-adrenoceptor blockade on glucocorticoid-induced memory retrieval deficits. Am J Psychiatry 164:967-969.

Devauges V, Sara SJ (1991) Memory retrieval enhancement by locus coeruleus stimulation: evidence for mediation by $\beta$-receptors. Behav Brain Res 43:93-97.

Dudai Y, Eisenberg M (2004) Rites of passage of the engram: reconsolidation and the lingering consolidation hypothesis. Neuron 44:93-100.

Duvarci S, Mamou CB, Nader K (2006) Extinction is not a sufficient condition to prevent fear memories from undergoing reconsolidation in the basolateral amygdala. Eur J Neurosci 24:249-260.

Fallon JH, Koziell DA, Moore RY (1978) Catecholamine innervation of the basal forebrain. II. Amygdala, suprarhinal cortex and entorhinal cortex. J Comp Neurol 180:509-532.

Fenker DB, Schott BH, Richardson-Klavehn A, Heinze HJ, Düzel E (2005) Recapitulating emotional context: activity of amygdala, hippocampus and fusiform cortex during recollection and familiarity. Eur J Neurosci 21:1993-1999.

Fischer H, Wright CI, Whalen PJ, McInerney SC, Shin LM, Rauch SL (2003) Brain habituation during repeated exposure to fearful and neutral faces: a functional MRI study. Brain Res Bull 59:387-392.

Kensinger EA, Corkin S (2004) Two routes to emotional memory: distinct neural processes for valence and arousal. Proc Natl Acad Sci U S A 101:3310-3315.

Kindt M, Soeter M, Vervliet B (2009) Beyond extinction: erasing human fear responses and preventing the return of fear. Nat Neurosci 12: $256-258$.

LeDoux JE (2000) Emotion circuits in the brain. Annu Rev Neurosci 23:155-184.

Maratos EJ, Dolan RJ, Morris JS, Henson RN, Rugg MD (2001) Neural activity associated with episodic memory for emotional context. Neuropsychologia 39:910-920.

Marr D (1970) A theory for cerebral neocortex. Proc R Soc Lond B Biol Sci 176:161-234.

McGaugh JL (2000) Memory-a century of consolidation. Science 287: $248-251$.

McGaugh JL (2004) The amygdala modulates the consolidation of memories of emotionally arousing experiences. Annu Rev Neurosci 27:1-28.

McGaugh JL, McIntyre CK, Power AE (2002) Amygdala modulation of memory consolidation: interaction with other brain systems. Neurobiol Learn Mem 78:539-552.

Mickley Steinmetz KR, Kensinger EA (2009) The effects of valence and arousal on the neural activity leading to subsequent memory. Psychophysiology 46:1190-1199.

Morris CD, Bransford JD, Franks JJ (1977) Levels of processing versus transfer appropriate processing. J Verbal Learn Verbal Behav 16:519-533.

Murchison CF, Zhang X-Y, Zhang W-P, Ouyang M, Lee A, Thomas SA (2004) A distinct role for norepinephrine in memory retrieval. Cell 117:131-143.

Nadel L, Land C (2000) Memory traces revisited. Nat Rev Neurosci 1:209-212.

Nadel L, Moscovitch M (1997) Memory consolidation, retrograde amnesia and the hippocampal complex. Curr Opin Neurobiol 7:217-227.

Nader K, Schafe GE, Le Doux JE (2000) Fear memories require protein synthesis in the amygdala for reconsolidation after retrieval. Nature 406:722-726.

Phelps EA, O'Connor KJ, Gatenby JC, Gore JC, Grillon C, Davis M (2001) Activation of the left amygdala to a cognitive representation of fear. Nat Neurosci 4:437-441.

Pickel VM, Segal M, Bloom FE (1974) A radioautographic study of the efferent pathways of the nucleus locus coeruleus. J Comp Neurol 155:15-42.

Pitman RK, Sanders KM, Zusman RM, Healy AR, Cheema F, Lasko NB, Cahill L, Orr SP (2002) Pilot study of secondary prevention of posttraumatic stress disorder with propranolol. Biol Psychiatry 51:189-192.

Ressler KJ, Mayberg HS (2007) Targeting abnormal neural circuits in mood and anxiety disorders: from the laboratory to the clinic. Nat Neurosci 10:1116-1124.

Richardson MP, Strange BA, Dolan RJ (2004) Encoding of emotional memories depends on amygdala and hippocampus and their interactions. Nat Neurosci 7:278-285.

Sara SJ, Devauges V (1988) Priming stimulation of locus coeruleus facilitates memory retrieval in the rat. Brain Res 438:299-303.

Sara SJ, Devauges V (1989) Idazoxan, an $\alpha$-2 antagonist, facilitates memory retrieval in the rat. Behav Neural Biol 51:401-411.

Seidenbecher T, Laxmi TR, Stork O, Pape H-C (2003) Amygdalar and hippocampal theta rhythm synchronization during fear memory retrieval. Science 301:846-850.

Smith AP, Henson RN, Dolan RJ, Rugg MD (2004) fMRI correlates of the episodic retrieval of emotional contexts. Neuroimage 22:868-878.

Smith AP, Stephan KE, Rugg MD, Dolan RJ (2006) Task and content modulate amygdala-hippocampal connectivity in emotional retrieval. Neuron 49:631-638.

Spielberger C, Gorsuch RL, Lushene R, Vagg PR, Jacobs GA (1983) Manual for the state-trait anxiety scale. Palo Alto, CA: Consulting Psychologists.

Sterpenich V, D’Argembeau A, Desseilles M, Balteau E, Albouy G, Vandewalle G, Degueldre C, Luxen A, Collette F, Maquet P (2006) The locus ceruleus is involved in the successful retrieval of emotional memories in humans. J Neurosci 26:7416-7423.

Strange BA, Dolan RJ (2001) Adaptive anterior hippocampal responses to oddball stimuli. Hippocampus 11:690-698.

Strange BA, Dolan RJ (2004) $\beta$-Adrenergic modulation of emotional memory-evoked human amygdala and hippocampal responses. Proc Natl Acad Sci U S A 101:11454-11458.

Strange BA, Hurlemann R, Dolan RJ (2003) An emotion-induced retrograde amnesia in humans is amygdala- and $\beta$-adrenergic-dependent. Proc Natl Acad Sci U S A 100:13626-13631.

Tollenaar MS, Elzinga BM, Spinhoven P, Everaerd W (2009) Immediate and prolonged effects of cortisol, but not propranolol, on memory retrieval in healthy young men. Neurobiol Learn Mem 91:23-31.

Vaiva G, Ducrocq F, Jezequel K, Averland B, Lestavel P, Brunet A, Marmar CR (2003) Immediate treatment with propranolol decreases posttraumatic stress disorder two months after trauma. Biol Psychiatry 54:947-949.

Zhang W-P, Guzowski JF, Thomas SA (2005) Mapping neuronal activation and the influence of adrenergic signaling during contextual memory retrieval. Learn Mem 12:239-247. 